\title{
Some Progress on Total Bondage in Graphs
}

\author{
Nader Jafari Rad • Joanna Raczek
}

Received: 25 August 2011 / Revised: 12 February 2013 / Published online: 17 March 2013

(C) The Author(s) 2013. This article is published with open access at Springerlink.com

\begin{abstract}
The total bondage number $b_{t}(G)$ of a graph $G$ with no isolated vertex is the cardinality of a smallest set of edges $E^{\prime} \subseteq E(G)$ for which (1) $G-E^{\prime}$ has no isolated vertex, and (2) $\gamma_{t}\left(G-E^{\prime}\right)>\gamma_{t}(G)$. We improve some results on the total bondage number of a graph and give a constructive characterization of a certain class of trees achieving the upper bound on the total bondage number.
\end{abstract}

Keywords Domination number $\cdot$ Total domination $\cdot$ Total bondage $\cdot$ Tree

Mathematics Subject Classification (2000) $\quad$ 05C69 • 05C05

\section{Introduction}

Let $G=(V(G), E(G))$ be a simple graph of order $n$. We denote the open neighborhood of a vertex $v$ of $G$ by $N_{G}(v)$ or just $N(v)$, and its closed neighborhood by $N_{G}[v]=N[v]$. For a vertex set $S \subseteq V(G), N(S)=\bigcup_{v \in S} N(v)$ and $N[S]$ $=\bigcup_{v \in S} N[v]$. The degree $\operatorname{deg}_{G}(x)$ (or just $\operatorname{deg}(x)$ ) of a vertex $x$ denotes the number of neighbors of $x$ in $G$. The maximum and minimum degree of a vertex of $G$ are denoted by $\Delta(G)$ and $\delta(G)$, respectively. A set of vertices $S$ in $G$ is a dominating set

\footnotetext{
N. J. Rad

Department of Mathematics, Shahrood University of Technology, Shahrood, Iran e-mail: n.jafarirad@shahroodut.ac.ir

J. Raczek $(\varangle)$

Department of Applied Physics and Mathematics, Gdansk University of Technology, Narutowicza 11/12, Gdańsk 80-233, Poland e-mail: Joanna.Raczek@pg.gda.pl
} 
if $N[S]=V(G)$. The domination number of $G$, denoted by $\gamma(G)$, is the minimum cardinality of a dominating set of $G$. A set of vertices $S$ in a graph $G$ without isolated vertex is a total dominating set, or just TDS, if $N(S)=V(G)$. The total domination number of $G$, denoted by $\gamma_{t}(G)$, is the minimum cardinality of a TDS of $G$. We refer to a $\gamma_{t}(G)$-set in a graph $G$ as a minimum cardinality TDS of $G$. For references on domination in graphs see for example [6,7].

With $K_{n}$ we denote the complete graph on $n$ vertices, with $P_{n}=\left(v_{1}, v_{2}, \ldots, v_{n}\right)$ the path on $n$ vertices, with $C_{n}$ the cycle of length $n$, and with $K_{p, q}$ the complete bipartite graph, which one partite set is of cardinality $p$ and another partite set is of cardinality $q$. We also let $g(G)$ be the girth of $G$, that is the length of a shortest cycle in $G$. Denote by $d_{G}(u, v)$ the distance between $u$ and $v$ in $G$. The diameter of $G$ is defined as $\operatorname{diam}(G)=\max \left\{d_{G}(u, v): u, v \in V(G)\right\}$.

If $S$ is a subset of $V(G)$, then we denote by $G[S]$ the subgraph of $G$ induced by $S$. We recall that a leaf in a graph is a vertex of degree one and a support vertex is one that is adjacent to a leaf. Let $S(G)$ be the set of all support vertices in a graph $G$. A pendant edge is an edge incident with a leaf.

The bondage number $b(G)$ of a nonempty graph $G$ is the minimum cardinality among all sets of edges $E^{\prime} \subseteq E(G)$ for which $\gamma\left(G-E^{\prime}\right)>\gamma(G)$. This concept was introduced by Bauer, Harary, Nieminen and Suffel in [1], and has been further studied for example in $[4,5,13]$. For more information on this topic we refer the reader to the survey article by Dunbar, Haynes, Teschner and Volkmann [3].

Kulli et al. in [9] introduced the concept of total bondage in graphs. The total bondage number $b_{t}(G)$ of a graph $G$ with no isolated vertex is the cardinality of a smallest set of edges $E^{\prime} \subseteq E(G)$ for which (1) $G-E^{\prime}$ has no isolated vertex, and (2) $\gamma_{t}\left(G-E^{\prime}\right)>\gamma_{t}(G)$. In the case that there is no subset of edges $E^{\prime}$ such that (1) and (2) both hold, we define $b_{t}(G)=\infty$. The total bondage number was further studied in [12]. Other types of bondage in trees give raise to many interesting problems. This was studied for example in [10] and [11].

In this paper we continue the study of total bondage in graphs. Since determining the exact number of the total bondage number for general graphs is a hard problem [8], in this paper we establish bounds on this number in some classes of graphs. In Sect. 2 we state some known and preliminary results. In Sect. 3 we first obtain an improved upper bound for the total bondage number of a tree and then we give an interesting constructive characterization of a certain class of trees achieving equality for the upper bound. In Sect. 4 we obtain some upper bounds on $b_{t}(G)$ in terms of maximum and minimum degrees, which in particular improve similar previous bounds.

\section{Known and Preliminary Results}

In this section we give some known and preliminary results which we use in the next sections. We begin with the following exact values of total bondage number of paths and cycles. 
Proposition 1 (Kulli et al. [9]) For $n \geq 2$,

$$
b_{t}\left(P_{n}\right)=\left\{\begin{array}{ll}
\infty & \text { if } n \leq 3 \\
1 & \text { if } n \geq 4, n \neq 2(\bmod 4) \\
2 & \text { if } n \geq 4, n \equiv 2(\bmod 4)
\end{array} .\right.
$$

Proposition 2 (Kulli et al. [9]) For $n \geq 3$,

$$
b_{t}\left(C_{n}\right)= \begin{cases}\infty & \text { if } n=3 \\ 2 & \text { if } n \geq 4, n \neq 2(\bmod 4) \\ 3 & \text { if } n \geq 4, n \equiv 2(\bmod 4)\end{cases}
$$

Sridharan et al. in [12] obtained the following upper bounds for the total bondage number of graphs.

Theorem 1 (Sridharan et al. [12]) Let $G$ be a connected graph of order $n \geq 4$. Then,

1. $b_{t}(G) \leq n-1$ if $g(G) \geq 5$,

2. $b_{t}(G) \leq n-2$ if $g(G)=4$,

3. $b_{t}(G) \leq n-2$ if there is a triangle which at least one of its vertices is a support vertex in $G$,

4. $b_{t}(G) \leq n-1$ if there is a triangle which at least one of its vertices is of degree two in $G$.

Theorem 2 (Sridharan et al. [12]) If $T$ is a tree on $n$ vertices and $T \neq K_{1, n-1}$, then

$$
b_{t}(T) \leq \min \left\{\Delta(T), \frac{n-1}{3}\right\} .
$$

We shall improve Theorems 1 and 2 . The following observations are easily verified.

Remark 1 If $p$ non-pendant edges can be removed from a graph $G$ to obtain a graph $H$ without an isolate vertex and with $b_{t}(H)=t$, then $b_{t}(G) \leq p+t$.

Remark 2 The total bondage number of a graph $G$ does not change if we add a leaf to a support vertex of $G$.

Remark 3 Let $x$ be a support vertex of a graph $G$ adjacent to at least two leaves. Then the total bondage number of $G$ does not change if we remove from $G$ a leaf adjacent to $x$.

The following is a direct consequence of Remark 2.2 of [12].

Proposition 3 For a graph $G, b_{t}(G)=\infty$ if and only if each connected component of $G$ is either $C_{3}$ or contains only pendant edges.

Let $\mathcal{B}$ be the class of all graphs $G$ with no isolated vertex such that $b_{t}(G) \neq \infty$. From now on all graphs $G$ considered in the rest of the paper belong to $\mathcal{B}$. 


\section{Trees}

In this section we study total bondage number of trees $T \in \mathcal{B}$. We improve a previous upper bound for the total bondage number of a tree. Then we present a constructive characterization for a certain class of trees achieving equality for the upper bound. Some of the results presented in this section reference to lemmas presented in Sect. 4. First we have the following upper bound for caterpillars. We recall that a caterpillar is a tree with the property that the removal of its leaves results in a path.

Proposition 4 For a caterpillar $T, b_{t}(T) \leq 2$.

Proof Let $T$ be a caterpillar. Since $T \in \mathcal{B}, \operatorname{diam}(T) \geq 3$. If $\operatorname{diam}(T) \in\{3,4\}$, then obviously $b_{t}(T)=1$. So assume that $\operatorname{diam}(T) \geq 5$. Let $\left(x, x_{1}, x_{2}, x_{3}, \ldots, x_{\operatorname{diam}(T)}\right)$ be a diametrical path, where $x$ is a leaf. Surely $\gamma_{t}\left(T-\left\{x_{1} x_{2}, x_{3} x_{4}\right\}\right)>\gamma_{t}(T)$ and the result follows.

If $T$ is a tree with maximum degree two, then by Proposition $1, b_{t}(T) \leq 2$. In the following we give a sharp upper bound for the total bondage number of trees with maximum degree at least three.

Theorem 3 For any tree $T$ with maximum degree at least three,

$$
b_{t}(T) \leq \Delta(T)-1 \text {. }
$$

Proof Let $T$ be a tree on $n$ vertices, and let $\Delta(T) \geq 3$. By Remarks 2 and 3, in what follows, we simply consider only trees in which every support vertex is adjacent to exactly one leaf. Since $T \in \mathcal{B}, \operatorname{diam}(T) \geq 3$. If $\operatorname{diam}(T)=3$, then obviously $b_{t}(T)=1$. So assume that $\operatorname{diam}(T) \geq 4$. Let $P=\left(x, x_{1}, x_{2}, x_{3}, \ldots, x_{\operatorname{diam}(T)}\right)$ be a diametrical path. Note that $x$ is a leaf. Suppose to the contrary that $b_{t}(T) \geq \Delta(T)$. Since $b_{t}(T) \geq 3, \gamma_{t}\left(T-x_{1} x_{2}\right)=\gamma_{t}(T)$. This implies that for any mimimum TDS $S$ of $T-x_{1} x_{2}, x_{2} \notin S$. As a consequent $\operatorname{deg}\left(x_{2}\right)=2$, since $P$ is the longest path of $T$. By Lemma 7, $\operatorname{deg}\left(x_{3}\right) \geq 3$. If $\operatorname{diam}(T)=4$, then $\gamma_{t}(T)=3$, and $\gamma_{t}\left(T-x_{1} x_{2}\right)=4$, which contradicts $b_{t}(T) \geq \Delta(T)$. So we suppose that $\operatorname{diam}(T) \geq 5$. We consider the following cases.

1. $x_{3}$ is a support vertex. Let $A$ be the set of all pendant edges incident with $x_{3}$. Let $T_{1}$ be a graph obtained from $T-x_{1} x_{2}$ by removing each edge incident with $x_{3}$ with exception of $x_{2} x_{3}$ and the edges in $A$. Let $H_{1}$ be the component of $T_{1}$ containing $x_{3}$. It is obvious that $H_{1}$ is a star. Let $S$ be a $\gamma_{t}\left(T_{1}\right)$-set, and let $S_{1}=S \cap V\left(H_{1}\right)$. It follows that $\left(S-\left(S_{1} \cup\{x\}\right)\right) \cup\left\{x_{2}, x_{3}\right\}$ is a TDS for $T$ of cardinality less than $\gamma_{t}(T)$, a contradiction.

2. $x_{3}$ is not a support vertex. First suppose that there is a support vertex $y_{1} \neq x_{4}$ where $y_{1}$ is adjacent to $x_{3}$. Let $y_{2}$ be the leaf adjacent to $y_{1}$. If $y_{1}$ is adjacent to a support vertex $y_{3}$ and $y_{4}$ is a leaf adjacent to $y_{3}$, then the path $P^{\prime}=$ $\left(y_{4}, y_{3}, y_{1}, x_{3}, x_{4}, \ldots, x_{\operatorname{diam}(T)}\right)$ is a diametrical path. Then similar to what we observed for $\operatorname{deg}\left(x_{2}\right)$, we obtain $\operatorname{deg}\left(y_{1}\right)=2$, a contradiction. So any vertex of $N\left(y_{1}\right)-\left\{x_{3}\right\}$ is a leaf. Let $S_{2}$ be a $\gamma_{t}\left(T-\left\{x_{3} y_{1}, x_{1} x_{2}\right\}\right)$-set. Since $x_{2}$ is a leaf in 
Fig. 1 Tree $R_{5}$

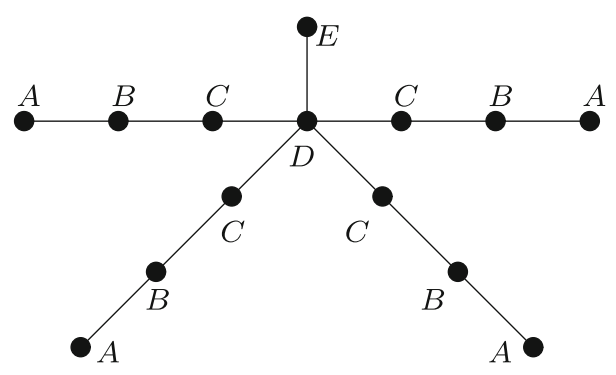

$T-\left\{x_{3} y_{1}, x_{1} x_{2}\right\}$, we have $x_{3} \in S_{2}$. However, $\gamma_{t}\left(T-\left\{x_{3} y_{1}, x_{1} x_{2}\right\}\right)=\gamma_{t}(T)$. Let $T_{2}$ be the component of $T-\left\{x_{3} y_{1}, x_{1} x_{2}\right\}$ that contains $y_{1}$. Let $v \neq y_{1}$ be a vertex such that $v \in V\left(T_{2}\right) \cap S_{2}$. Then $\left(S_{2}-\{x, v\}\right) \cup\left\{x_{2}\right\}$ is a TDS for $T$ of cardinality less than $\gamma_{t}(T)$. This contradiction implies that no vertex in $N\left(x_{3}\right)-\left\{x_{4}\right\}$ is a support vertex. Let $T_{3}$ be the component of $T-x_{3} x_{4}$ containing $x_{3}$, and let $T_{4}$ be obtained from $T_{3}$ by removing all leaves of $T_{3}$. So any leaf of $T_{4}$ is at distance two from $x_{3}$. Furthermore, with a similar discussion as in the proof for $\operatorname{deg}\left(x_{2}\right)=2$, we observe that $T_{4}$ is a tree obtained from $K_{1, \operatorname{deg}\left(x_{3}\right)-1}$ by subdividing any edge. It is obvious that $\gamma_{t}\left(T-\left\{x_{1} x_{2}, x_{3} x_{4}\right\}\right)=\gamma_{t}(T)$. Let $S_{3}$ be a $\gamma_{t}\left(T-\left\{x_{1} x_{2}, x_{3} x_{4}\right\}\right)$-set containing as small number of leaves as possible. Then $x_{3} \in S_{3}$, since $x_{2}$ is a leaf in $T-\left\{x_{1} x_{2}, x_{3} x_{4}\right\}$. Further, $V\left(T_{4}\right) \subseteq S_{3}$. Now $\left(S_{3}-\left\{x, x_{3}\right\}\right) \cup\left\{x_{2}\right\}$ is a TDS for $T$ of cardinality less than $\gamma_{t}(T)$, a contradiction.

We note that Theorem 3 improves Theorem 2 in the case when $\Delta(T)<\frac{n(T)+2}{3}$.

\subsection{Characterization of Extremal Trees}

In this subsection we obtain a constructive characterization for a certain class of trees achieving equality for the upper bound of Theorem 3 . We will characterize all trees $T_{k}$, where each edge is incident with a support vertex, with $k=\Delta(T) \geq 4$ and having $b_{t}(T)=\Delta-1$. By Remarks 2 and 3, in what follows, we simply consider only trees for which every support vertex is adjacent to exactly one leaf. Denote by $\mathcal{T}_{k}$ the set of all trees $T_{k}$ in which every support vertex is adjacent to exactly one leaf, each edge is incident with a support vertex and having $b_{t}(T)=k-1$, where $k=\Delta(T) \geq 4$.

In [12] a tree $H_{k}$ with $\Delta\left(H_{k}\right)=k+1$ and $b_{t}\left(H_{k}\right)=k$ was introduced as follows. Let $x$ be the central vertex of $K_{1, k-1}$ for some $k \geq 4$ and let $H_{k}$ be a tree obtained from $K_{1, k-1}$ by subdividing each edge twice and adding a new vertex $y$ and joining $y$ to $x$. (Note that $y$ is a leaf in $H_{k}$ and $x$ is a support vertex adjacent to $y$ ). In this paper we label $H_{k}$ with vertex labels $\{A, B, C, D, E\}$ to get a labeled tree $R_{k}$ as follows. Let $l(y)=E$, let each leaf except of $y$ has label $A$, let each support vertex of degree 2 has label $B$, let the vertex of degree $k-1$ has label $D$ and let every other vertex has label $C$. It is straightforward to see that $\gamma_{t}\left(R_{k}\right)=2 k-1, k=\Delta\left(R_{k}\right)$ and $b_{t}\left(R_{k}\right)=k-1=\Delta\left(R_{k}\right)-1$. Moreover each edge of $R_{k}$ is incident with a support vertex. See $R_{5}$ in Fig. 1 . 
We describe a procedure to build a family $\mathcal{R}_{k}$ of trees $T_{k}$ with vertex labels belonging to the set $\{A, B, C, D, E\}$ and with $k=\Delta\left(T_{k}\right) \geq 4$ as follows. Let $T_{k} \in \mathcal{R}_{k}$ and let $x \in V\left(T_{k}\right)$. We call $x$ an active vertex if $l(x)=D$ and there is a path $P_{3}$ attached to $x$. Let $\mathcal{R}_{k}$ be such that:

1. Contains $R_{k}$ for $k \geq 4$, described as above;

2. Is closed under the following three operations $\mathcal{O}_{1}, \mathcal{O}_{2}, \mathcal{O}_{3}$ :

- Operation $\mathcal{O}_{1}$. Assume $T_{k}^{1}$ and $T_{k}^{2}$ are two trees belonging to $\mathcal{R}_{k}$. Let $x^{1}$ and $x^{2}$ be active vertices belonging to $T_{k}^{1}$ and $T_{k}^{2}$, respectively and let $P_{3}^{1}$ and $P_{3}^{2}$ be the paths on three vertices attached to $x^{1}$ and $x^{2}$, respectively. Then remove $P_{3}^{1}$ and $P_{3}^{2}$ and add the edge $x^{1} x^{2}$ to obtain a new tree with maximum degree $k$.

- Operation $\mathcal{O}_{2}$. Assume $T_{k}^{1}$ and $T_{k}^{2}$ are two trees belonging to $\mathcal{R}_{k}$ and let $x_{1}^{1}, x_{1}^{2}$ be vertices with label $C$ belonging to $T_{k}^{1}, T_{k}^{2}$, respectively, such that $d_{T_{k}^{1}}\left(x_{1}^{1}\right)+d_{T_{k}^{2}}\left(x_{1}^{2}\right)-1 \leq k$ and $d_{T_{k}^{1}}\left(x_{2}^{1}\right)+d_{T_{k}^{2}}\left(x_{2}^{2}\right)-2 \leq k$, where $l\left(x_{2}^{1}\right)=$ $l\left(x_{2}^{2}\right)=B$ and $x_{2}^{1}$ is adjacent to $x_{1}^{1}$ and $x_{2}^{2}$ is adjacent to $x_{1}^{2}$. Denote by $x_{3}^{1}$ and $x_{3}^{2}$ the leaves adjacent to $x_{2}^{1}$ and $x_{2}^{2}$, respectively. Identify vertices $x_{j}^{1}$ and $x_{j}^{2}$ into one vertex $x_{j}$ for each $j \in\{1,2,3\}$ to obtain a new tree with maximum degree $k$. Let $l\left(x_{1}\right)=C, l\left(x_{2}\right)=B$ and $l\left(x_{3}\right)=A$.

- Operation $\mathcal{O}_{3}$. Assume $T_{k}^{1}$ and $T_{k}^{2}$ are two trees belonging to $\mathcal{R}_{k}$ and let $x_{1}^{1}, x_{1}^{2}$ be vertices with label $B$ belonging to $T_{k}^{1}, T_{k}^{2}$, respectively, such that $d_{T_{k}^{1}}\left(x_{1}^{1}\right)+d_{T_{k}^{2}}\left(x_{1}^{2}\right)-1 \leq k$. Denote by $x_{2}^{1}$ and $x_{2}^{2}$ the leaves adjacent to $x_{1}^{1}$ and $x_{1}^{2}$, respectively. Identify vertices $x_{j}^{1}$ and $x_{j}^{2}$ into one vertex $x_{j}$ for each $j \in$ $\{1,2\}$ to obtain a new tree with maximum degree $k$. Let $l\left(x_{1}\right)=B, l\left(x_{2}\right)=A$.

We first prove that $b_{t}\left(T_{k}\right)=\Delta\left(T_{k}\right)-1$ for each tree $T_{k}$ belonging to $\mathcal{R}_{k}$. To this aim we first make some observations, which follow immediately from the way in which each tree in the family $\mathcal{R}_{k}$ is constructed.

Remark 4 If $T_{k} \in \mathcal{R}_{k}$ and $v \in V\left(T_{k}\right)$, then

1. $l(v) \in\{A, E\}$ if and only if $v$ is a leaf;

2. $l(v) \in\{B, D\}$ if and only if $v$ is a support vertex;

3. If $l(v)=B$, then exactly one neighbour of $v$ has label $A$ and every other neighbour of $v$ has label $C$;

4. If $l(v)=C$, then each neighbour of $v$ is a support vertex. Moreover, exactly one neighbour of $v$ has label $B$ and every other neighbour of $v$ has label $D$;

5. If $l(v)=D$, then exactly one neighbour of $v$ has label $E$ and every other neighbour of $v$ has label $C$ or $D$. Moreover, $v$ has $k$ neighbours altogether;

6. Each edge of $T_{k}$ is incident with a support vertex, e.g. a vertex with label $B$ or $D$;

7. If $l(v) \in\{B, D\}$, then $v$ belongs to every minimum TDS of $T_{k}$;

8. If $l(v) \in\{A, C\}$, then $v$ belongs to some minimum TDS of $T_{k}$ and each neighbour of $v$ belongs to every minimum TDS of $T_{k}$;

9. If $l(v)=E$, then $v$ belongs to no minimum TDS of $T_{k}$;

10. $\gamma_{t}\left(T_{k}\right)=2\left|\left\{u \in V\left(T_{k}\right): l(u)=B\right\}\right|+\left|\left\{u \in V\left(T_{k}\right): l(u)=D\right\}\right|$.

Lemma 1 For $k \geq 4$, if a tree $T_{k}$ belongs to the family $\mathcal{R}_{k}$, then $T_{k}$ without labels on vertices belongs to $\mathcal{T}_{k}$. 
Proof Let $T_{k}$ be a tree belonging to the family $\mathcal{R}_{k}$. Clearly $\Delta\left(T_{k}\right)=k$ and each edge of $T_{k}$ is incident with a support vertex, so it suffices to justify that $b_{t}\left(T_{k}\right)=k-1$. Suppose $b_{t}\left(T_{k}\right)<k-1$. Let $F \subseteq E\left(T_{k}\right)$ be such that $\gamma_{t}\left(T_{k}\right)<\gamma_{t}\left(T_{k}-F\right)$ and $|F|=b_{t}\left(T_{k}\right) \leq k-2$. By Remark 4, $F$ contains only edges $v w$ of three types:

$-l(v)=l(w)=D ;$

$-l(v)=B$ and $l(w)=C$;

- $l(v)=C$ and $l(w)=D$.

For any $v w \in F$ since $|F|=b_{t}\left(T_{k}\right) \leq k-2$, we conclude that $\gamma_{t}\left(T_{k}-(F\right.$ $-\{v w\}))=\gamma_{t}\left(T_{k}\right)$ and $\gamma_{t}\left(T_{k}-F\right)>\gamma_{t}\left(T_{k}-(F-\{v w\})\right)$. We consider three cases for labels of $v$ and $w$.

Case 1. Assume first that $l(v)=l(w)=D$. Remark 4 implies that both $v$ and $w$ have in $T_{k}-(F-\{v w\})$ at least two neighbours with labels in $\{C, D\}$ such that if a vertex has label $C$, then is adjacent to a support vertex with label $B$ and if a vertex has label $D$, then it is adjacent to a vertex with label $C$ or $D$, which is not a leaf. Thus, $\gamma_{t}\left(T_{k}-F\right)=\gamma_{t}\left(T_{k}-(F-\{v w\})\right)$, which is impossible.

Case 2. Assume now that $l(v)=B$ and $l(w)=C$. Since $|F|=b_{t}\left(T_{k}\right) \leq k-2$, we conclude that $\gamma_{t}\left(T_{k}-(F-\{v w\})\right)=\gamma_{t}\left(T_{k}\right)$ and $\gamma_{t}\left(T_{k}-F\right)>\gamma_{t}\left(T_{k}-(F-\{v w\})\right)$. Since $v w$ is a non-pendant edge, Remark 4 implies that $w$ is adjacent in $T_{k}-(F-\{v w\})$ to at least one vertex with label $D$, say $y$, such that $y$ has at least one neighbour in $T_{k}-(F-\{v w\})$, except of $w$, with label in $\{C, D\}$ such that if it has label $C$, then it is adjacent to a support vertex with label $B$ and if a vertex has label $D$, then it is adjacent to a vertex with label $C$ or $D$, which is not a leaf. Thus, $\gamma_{t}\left(T_{k}-F\right)=\gamma_{t}\left(T_{k}-(F-\{v w\})\right)$, which is impossible.

Case 3. Lastly, assume that $l(v)=C$ and $l(w)=D$. Since $|F|=b_{t}\left(T_{k}\right) \leq k-2$, we conclude that $\gamma_{t}\left(T_{k}-(F-\{v w\})\right)=\gamma_{t}\left(T_{k}\right)$ and $\gamma_{t}\left(T_{k}-F\right)>\gamma_{t}\left(T_{k}-(F-\{v w\})\right)$. Since $v w$ is a non-pendant edge, Remark 4 implies that $v$ is adjacent in $T_{k}-(F-\{v w\})$ to at least one vertex with label in $\{B, D\}$, say $y \neq w$, such that $y$ has at least one neighbour in $T_{k}-(F-\{v w\})$. Moreover, $w$ has at least one neighbour, different from $v$, with label in $\{C, D\}$ such that if a vertex has label $C$, then it is adjacent to a support vertex with label $B$ and if a vertex has label $D$, then is adjacent to a vertex with label $C$ or $D$, which is not a leaf. Thus, $\gamma_{t}\left(T_{k}-F\right)=\gamma_{t}\left(T_{k}-(F-\{v w\})\right)$, which is impossible.

We conclude that $b_{t}\left(T_{k}\right) \geq k-1$ and Theorem 3 implies the desired result.

Lemma 2 If every support vertex of a tree $T$ is adjacent to exactly one leaf, $\Delta(T) \geq 4$ and $b_{t}(T)=\Delta(T)-1$, then

(a) $\operatorname{deg}_{T}(x)=\operatorname{deg}_{T}(y)=\Delta(T)-1$ for each adjacent support vertices $x, y$;

(b) $d_{T}(x, y) \geq 3$ for each pair of support vertices $x$, y of degree 2 .

Proof (a) It is an immediate consequence of Lemma 4.

(b) Let $x, y$ be two support vertices of degree 2 and denote by $x^{\prime}, y^{\prime}$ the two leaves adjacent to $x$ and $y$, respectively. If $x$ and $y$ are adjacent, then $T$ is a path on 4 vertices, which is a contradiction. Thus suppose $d(x, y)=2$ and let $z$ be the vertex adjacent to both $x$ and $y$. Since $T$ is not a path, $\operatorname{deg}_{T}(z) \geq 3$. Let $S$ be a smallest TDS of $T-x z-y z$. Then $x, x^{\prime}, y, y^{\prime}$ belong to $S$, but on the other hand, $\left(S-\left\{x^{\prime}, y^{\prime}\right\}\right) \cup\{z\}$ is a smaller TDS of $T$. 
Lemma 3 If a tree $T_{k}$ belongs to the family $\mathcal{T}_{k}$ for some $k \geq 4$, then it is possible to label vertices of $T_{k}$ with labels $\{A, B, C, D, E\}$ in such a way $T_{k}$ belongs to the family $\mathcal{R}_{k}$.

Proof Let $T_{k}$ be a tree belonging to the family $\mathcal{T}_{k}$. Then, by the definition of $\mathcal{T}_{k}$, every support vertex of $T_{k}$ is adjacent to exactly one leaf, each edge of $T_{k}$ is incident with a support vertex and $b_{t}\left(T_{k}\right)=\Delta\left(T_{k}\right)-1=k-1$, where $k=\Delta\left(T_{k}\right) \geq 4$. Since $b_{t}\left(T_{k}\right)=$ $\Delta\left(T_{k}\right)-1 \geq 3$, clearly $\operatorname{diam}\left(T_{k}\right) \geq 4$. Let $P=\left(x, x_{1}, x_{2}, x_{3}, \ldots, x_{\operatorname{diam}\left(T_{k}\right)}\right)$ be a diametrical path. Since $\gamma_{t}\left(T_{k}-x_{1} x_{2}\right)=\gamma_{t}\left(T_{k}\right)$, we conclude that for any minimum TDS $S$ of $T_{k}-x_{1} x_{2}, x_{2} \notin S$. Since $P$ is a longest path of $T_{k}$ it follows that $\operatorname{deg}\left(x_{2}\right)=2$. By Lemma 7, $\operatorname{deg}\left(x_{3}\right) \geq 3$. If $\operatorname{diam}\left(T_{k}\right)=4$, then $\gamma_{t}\left(T_{k}\right)=3$ and $\gamma_{t}\left(T_{k}-x_{1} x_{2}\right)=4$, which contradicts $b_{t}\left(T_{k}\right)=\Delta\left(T_{k}\right)-1$. So we assume that $\operatorname{diam}\left(T_{k}\right) \geq 5$. Since each edge of $T_{k}$ is incident with a support vertex, we conclude that $x_{3}$ is a support vertex. Then by Lemma 5 ,

$$
\begin{aligned}
\Delta\left(T_{k}\right)-1= & k-1=b_{t}(G) \leq \operatorname{deg}\left(x_{1}\right)+\operatorname{deg}\left(x_{2}\right) \\
& +\operatorname{deg}\left(x_{3}\right)-5=2+2+\operatorname{deg}\left(x_{3}\right)-5 .
\end{aligned}
$$

Hence $\operatorname{deg}\left(x_{3}\right)=\Delta\left(T_{k}\right)$. Moreover, if there is a leaf at distance 3 from $x_{3}$, say $\left(z, z_{1}, z_{2}, x_{3}\right)$ is the path from the leaf $z \neq x$ to $x_{3}$, where $z_{2} \neq x_{2}$, then similar to what we observed for $x_{1}$ and $x_{2}$, we obtain $\operatorname{deg}_{T_{k}}\left(z_{1}\right)=\operatorname{deg}_{T_{k}}\left(z_{2}\right)=2$.

Suppose $x_{3}$ is adjacent to a support vertex, say $y^{\prime}$, and let $y^{\prime \prime}$ be the leaf adjacent to $y^{\prime}$. Then $\operatorname{deg}_{T_{k}}\left(y^{\prime}\right)=2$. Let $S$ be a minimum TDS of $T_{k}-x_{3} y^{\prime}$ containing as small number of leaves as possible. Then $S-\left\{y^{\prime \prime}\right\}$ is a TDS of $T_{k}$, implying $b_{t}\left(T_{k}\right)=1$, which is impossible. For the same reasons, $\operatorname{diam}\left(T_{k}\right) \geq 6$. Hence, the component of $T_{k}-x_{3} x_{4}$ containing $x_{3}$ is the tree $R_{k-1}$ and if $\operatorname{diam}\left(T_{k}\right)=6$, then $T_{k}=R_{k}$ and thus $T_{k} \in \mathcal{R}_{k}$. Therefore in what follows we assume $\operatorname{diam}\left(T_{k}\right) \geq 7$. We follow by induction on the number $s\left(T_{k}\right)$ of vertices of degree $\Delta\left(T_{k}\right)$ in $T_{k}$. If $s\left(T_{k}\right)=1$, then obviously $T_{k}=R_{k}$. Thus assume that for every tree $T_{k}^{\prime}$ with $s\left(T_{k}^{\prime}\right)<s\left(T_{k}\right)$, if $T_{k}^{\prime} \in \mathcal{T}_{k}$, then it is possible to label the vertices of $T_{k}^{\prime}$ in such a way $T_{k}^{\prime} \in \mathcal{R}_{k}$.

1. If $x_{4}$ is a support vertex, then by our assumptions, $x_{4}$ is adjacent to exactly one leaf and by Lemma 4 we obtain that $x_{4}$ is of degree $k$. Denote by $T^{x_{3}}$ and $T^{x_{4}}$ the two components of $T_{k}-x_{3} x_{4}$ containing $x_{3}$ and $x_{4}$, respectively. Attach $P_{3}$ to $x_{3}$ to obtain a tree $T_{k}^{x_{3}}$ and attach $P_{3}: q_{1}-q_{2}-q_{3}$ to $x_{4}$ to obtain a tree $T_{k}^{x_{4}}$. Clearly $T_{k}^{x_{3}}=R_{k}$ and $\Delta\left(T_{k}^{x_{4}}\right)=k$. Moreover, $b_{t}\left(T_{k}^{x_{3}}\right)=k-1$. Suppose $b_{t}\left(T_{k}^{x_{4}}\right)<$ $k-1$. In this situation let $F \subseteq E\left(T_{k}^{x_{4}}\right)$ be such that $\gamma_{t}\left(T_{k}^{x_{4}}\right)<\gamma_{t}\left(T_{k}^{x_{4}}-F\right)$ and $|F|=b_{t}\left(T_{k}^{x_{4}}\right) \leq k-2$.

If $x_{4} q_{3} \in F$, then for $F^{\prime}=\left(F-\left\{x_{4} q_{3}\right\}\right) \cup\left\{x_{3} x_{4}\right\}$ we would obtain $\gamma_{t}\left(T_{k}\right)<$ $\gamma_{t}\left(T_{k}-F^{\prime}\right)$ contradicting that $b_{t}\left(T_{k}\right)=k-1$. Thus $x_{4} q_{3} \notin F$.

If $q_{2} q_{3} \in F$ and $x_{4} q_{3} \notin F$, then since $x_{4}$ is a neighbour of at least two leaves in $T_{k}^{x_{4}}-F$, Remark 3 implies that $b_{t}\left(T_{k}^{x_{4}}-F\right)=b_{t}\left(\left(T_{k}^{x_{4}}-F\right)-q_{3}\right)$. Hence for $F^{\prime \prime}=\left(F-\left\{q_{2} q_{3}\right\}\right) \cup\left\{x_{4} q_{3}\right\}$ we would have $\gamma_{t}\left(T_{k}^{x_{4}}\right)<\gamma_{t}\left(T_{k}^{x_{4}}-F^{\prime \prime}\right)$ and again we would obtain a contradiction.

If $q_{2} q_{3} \notin F$ and $x_{4} q_{3} \notin F$, then clearly $\gamma_{t}\left(T_{k}\right)<\gamma_{t}\left(T_{k}-F\right)$, which is impossible. Therefore, $b_{t}\left(T_{k}^{x_{4}}\right)=k-1$ and by induction hypothesis, $T_{k}^{x_{4}}$ is in $\mathcal{R}_{k}$. Since $x_{4}$ is 
an active vertex in $T_{k}^{x_{4}}$ and $x_{3}$ is an active vertex in $T_{k}^{x_{3}}$, we conclude that $T_{k}$ may be obtained from $T_{k}^{x_{3}}$ and $T_{k}^{x_{4}}$ by Operation $\mathcal{O}_{1}$.

2. If $x_{4}$ is not a support vertex, then each neighbour of $x_{4}$ is a support vertex since each edge is incident with a support vertex. Thus, $x_{5}$ is a support vertex and let $x_{5}^{\prime}$ be the leaf adjacent to $x_{5}$.

(a) Assume additionally $x_{4}$ is a neighbour of a support vertex of degree 2, say $y$, and let $y^{\prime}$ be the leaf adjacent to $y$. Let $\operatorname{deg}_{T_{k}}\left(x_{4}\right) \geq 4$ and denote by $z$ a neighbour of $x_{4}$ different from $x_{3}, x_{5}, y$. Since $x_{4} z$ is incident with a support vertex, we obtain that $z$ is a support vertex. Denote by $z^{\prime}$ the leaf adjacent to $z$. If $\operatorname{deg}_{T_{k}}(z)=2$, then by Lemma $2, b_{t}\left(T_{k}\right)<3$. Hence $x_{4}$ is a neighbour of exactly one support vertex of degree 2 .

Thus assume $\operatorname{deg}_{T_{k}}(z) \geq 3$. Then by Lemma 2, each neighbour of $z$ is not a support vertex and $z$ belongs to a longest path of $T_{k}$. Moreover, denote by $S$ a minimum total dominating set of $T-\left(A \cup\left\{x_{4} y\right\}\right)$, where $A$ is a set of all nonpendant edges incident with $z$ except for $z x_{4}$. Without loss of generality we may assume that $z, x_{4}, y, y^{\prime}$ belong to $S$. Further, $S-\left\{y^{\prime}\right\}$ is a smaller TDS of $T_{k}$, so $b_{t}\left(T_{k}\right) \leq|A|+1$. Since $|A|=\operatorname{deg}_{T_{k}}(z)-2$ and $b_{t}\left(T_{k}\right)=\Delta\left(T_{k}\right)-1$, we conclude that $\operatorname{deg}_{T_{k}}(z)=\Delta\left(T_{k}\right)$. By similar reasoning, $\operatorname{deg}_{T_{k}}\left(x_{5}\right)=\Delta\left(T_{k}\right)$. Denote by $T^{z}$ and $T_{k}^{x_{4}}$ the two components of $T_{k}-z x_{4}$ containing $z$ and $x_{4}$, respectively. Attach $P_{3}$ to $z$ to obtain a tree $T_{k}^{z}$. Clearly $T_{k}^{z}=R_{k}$ and $\Delta\left(T_{k}^{x_{4}}\right)=k$. Moreover, $b_{t}\left(T_{k}^{z}\right)=k-1$.

Suppose $b_{t}\left(T_{k}^{x_{4}}\right)<k-1$. In this situation let $F \subseteq E\left(T_{k}^{x_{4}}\right)$ be such that $\gamma_{t}\left(T_{k}^{x_{4}}\right)<\gamma_{t}\left(T_{k}^{x_{4}}-F\right)$ and $|F|=b_{t}\left(T_{k}^{x_{4}}\right) \leq k-2$. Then $\gamma_{t}\left(T_{k}-(F \cup\right.$ $\left.\left.\left\{x_{4} z\right\}\right)\right)>\gamma_{t}\left(T_{k}-F\right)$, so $|F|=k-2$. Let $S$ be a minimum TDS of $T_{k}-F$. Since $x_{4}$ is not isolated in $T_{k}^{x_{4}}-F, x_{4}$ has at least two neighbours in $T_{k}-F$. Further, each such neighbour is a support vertex, so belongs to $S$. If $x_{4} y \in F$, then $x_{4} \notin S$ and $S$ is also a total dominating set of $T_{k}-\left(F \cup\left\{x_{4} z\right\}\right)$. If $x_{4} y \notin F$, then again $S$ is a TDS of $T_{k}-\left(F \cup\left\{x_{4} z\right\}\right)$. Thus $\gamma_{t}\left(T_{k}\right)=\gamma_{t}\left(T_{k}-F\right)=$ $\gamma_{t}\left(T_{k}-\left(F \cup\left\{x_{4} z\right\}\right)\right)$, which is impossible. Thus $b_{t}\left(T_{k}^{x_{4}}\right)=k-1$ and by induction hypothesis, $T_{k}^{x_{4}}$ is in $\mathcal{R}_{k}$. Let $l\left(y^{\prime}\right)=A, l(y)=B$ and $l\left(x_{4}\right)=C$. Hence $T_{k}$ may be obtained from $R_{k}$ and $T_{k}^{x_{4}}$ by Operation $\mathcal{O}_{2}$.

If $\operatorname{deg}_{T_{k}}\left(x_{4}\right)=3$, then we remove $x_{3} x_{4}$ to obtain trees $T^{x_{3}}$ and $T_{k}^{x_{4}}$ and the rest of the proof is similar to the case when $\operatorname{deg}_{T_{k}}\left(x_{4}\right) \geq 4$.

(b) If $x_{4}$ is not a neighbour of a support vertex of degree 2 and $\operatorname{deg}_{T_{k}}\left(x_{4}\right) \geq 3$. Then $x_{4}$ is a neighbour of a support vertex $z$, where $z \notin\left\{x_{3}, x_{5}\right\}$ and denote by $z^{\prime}$ the leaf adjacent to $z$. By similar arguing as above, we conclude that $\operatorname{deg}_{T_{k}}(z)=\Delta\left(T_{k}\right)$ and each neighbour of $z$ is not a support vertex and $z$ belongs to a longest path of $T_{k}$. Denote by $T^{z}$ and $T_{k}^{x_{4}}$ the two components of $T_{k}-x_{4} z$ containing $z$ and $x_{4}$, respectively. Attach $P_{3}$ to $z$ to obtain a tree $T_{k}^{z}$. Clearly $T_{k}^{z}=R_{k}$ and $\Delta\left(T_{k}^{x_{4}}\right)=k$. Moreover, $b_{t}\left(T_{k}^{z}\right)=k-1$. By similar arguments as in Case 2 a we conclude that $b_{t}\left(T_{k}^{x_{4}}\right)=k-1$ and by induction hypothesis, $T_{k}^{x_{4}}$ is in $\mathcal{R}_{k}$. Let $l\left(x_{5}^{\prime}\right)=A, l\left(x_{5}\right)=B$ and $l\left(x_{4}\right)=C$. Hence $T_{k}$ may be obtained from $R_{k}$ and $T_{k}^{x_{4}}$ by Operation $\mathcal{O}_{2}$.

(c) If $x_{4}$ is of degree 2, then denote by $T^{x_{4}}$ and $T_{k}^{x_{5}}$ the two components of $T_{k}-x_{4} x_{5}$ containing $x_{4}$ and $x_{5}$, respectively. Attach $P_{2}$ to $x_{4}$ to obtain a tree $T_{k}^{x_{4}}$. Clearly 


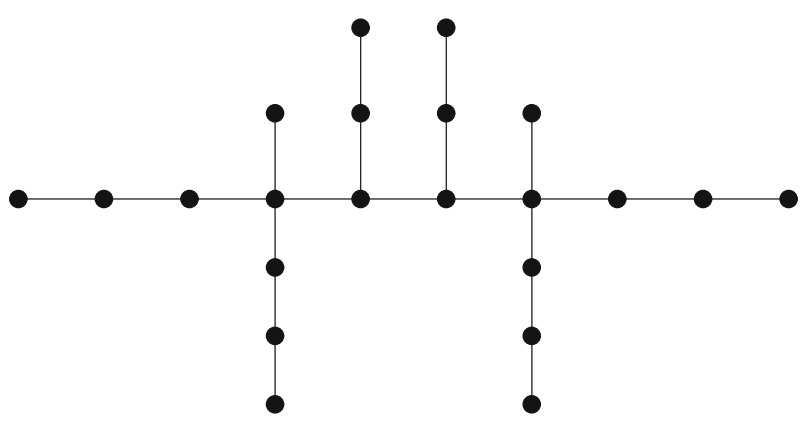

Fig. 2 Tree $T$

$T_{k}^{x_{4}}=R_{k}$ and $b_{t}\left(T_{k}^{x_{4}}\right)=k-1$. If $\Delta\left(T_{k}^{x_{5}}\right)<k$, then only $x_{3}$ and $x_{5}$ are vertices of degree $k$ in $T_{k}$ and $x_{5}$ has similar properties as $x_{3}$ and $\operatorname{diam}\left(T_{k}\right)=8$. However then $b_{t}\left(T_{k}\right)<k-1$, a contradiction. Thus $\Delta\left(T_{k}^{x_{5}}\right)=k$. By similar arguments as in Case 2a we conclude that $b_{t}\left(T_{k}^{x_{5}}\right)=k-1$ and by induction hypothesis, $T_{k}^{x_{5}}$ is in $\mathcal{R}_{k}$. Let $l\left(x_{5}^{\prime}\right)=A, l\left(x_{5}\right)=B$ and $l\left(x_{4}\right)=C$. Hence $T_{k}$ may be obtained from $R_{k}$ and $T_{k}^{x_{4}}$ by Operation $\mathcal{O}_{3}$.

As an immediate consequence of Lemmas 1 and 3, we have the following

Theorem 4 For each $k \geq 4$, if we omit vertex labelling of trees in $\mathcal{R}_{k}$,

$$
\mathcal{T}_{k}=\mathcal{R}_{k}
$$

In Fig. 2 is a tree $T$ with $b_{t}(T)=\Delta(T)-1=3$ and containing an edge not incident with a support vertex.

We finish this section with the following open problem.

conjecture $1 T$ is a tree with $\Delta(T) \geq 5$ and $b_{t}(T)=\Delta(T)-1$ if and only if $T$ belongs to the family $\mathcal{R}_{k}$ for $k \geq 5$.

\section{Upper Bounds}

In this section we obtain some upper bounds for the total bondage number of a graph $G \in \mathcal{B}$ in terms of maximum and minimum degrees.

Lemma 4 If $x$ and $y$ are two adjacent support vertices in a graph $G$, then

$$
b_{t}(G) \leq \min \{\operatorname{deg}(x), \operatorname{deg}(y)\}-1 .
$$

Proof Remove all edges incident with $x$ with exception of pendant edges to obtain a graph $H$. Suppose that $\gamma_{t}(H)=\gamma_{t}(G)$. Let $S$ be a $\gamma_{t}(H)$-set. Clearly, $y \in S$. Let $H_{1}$ be the component of $H$ containing $x$ and let $x_{1} \neq x$ be a vertex of $H_{1}$ with $x_{1} \in S$. Then $S-\left\{x_{1}\right\}$ is a TDS for $G$ of cardinality less than $\gamma_{t}(G)$, a contradiction. Therefore, $\gamma_{t}(H)>\gamma_{t}(G)$ and the result follows. 
Lemma 5 Let a graph $G$ contain a path $(x, y, z)$ on three vertices such that $\{x, z\} \subseteq$ $S(G)$ and $y \notin S(G)$, then

$$
b_{t}(G) \leq \operatorname{deg}(x)+\operatorname{deg}(y)+\operatorname{deg}(z)-5 .
$$

Proof Remove all edges incident with $x, y, z$ with exception of $x y$ and the pendant edges incident with $x$ or $z$, to obtain a graph $H$. It is obvious that $\gamma_{t}(H)>\gamma_{t}(H+y z) \geq$ $\gamma_{t}(G)$ and so the result follows. In case of $G=P_{5}$ the bound is sharp.

Similarly we have the following.

Lemma 6 If a graph $G$ contains a path $(x, y, z)$ on three vertices such that $\{x, y\} \cap$ $S(G)=\emptyset$ and $z \in S(G)$, then

$$
b_{t}(G) \leq \operatorname{deg}(x)+\operatorname{deg}(y)+\operatorname{deg}(z)-4 .
$$

Note that under assumptions of Lemma $5, b_{t}(G) \leq 3 \Delta(G)-5$ and under assumptions of Lemma $6, b_{t}(G) \leq 3 \Delta(G)-4$.

Theorem 5 Assume that a graph $G$ contains a path $\left(v_{1}, v_{2}, v_{3}, v_{4}\right)$ such that $G-\left\{v_{1}, v_{2}, v_{3}, v_{4}\right\}$ has no isolated vertex. If $G_{1}$ is the subgraph induced by $v_{1}, v_{2}, v_{3}$, $v_{4}$, then

$$
b_{t}(G) \leq \sum_{i=1}^{4} \operatorname{deg}\left(v_{i}\right)-\left|E\left(G_{1}\right)\right|-2
$$

This bound is sharp.

Proof Let $G_{1}$ be the subgraph induced by $v_{1}, v_{2}, v_{3}, v_{4}$. We remove all edges incident with $v_{1}, v_{2}, v_{3}, v_{4}$ except the edges $v_{1} v_{2}$ and $v_{3} v_{4}$ to obtain a graph $H$. If $\gamma_{t}(H)=$ $\gamma_{t}(G)$, then we let $S$ be a $\gamma_{t}(H)$-set. It follows that $S-\left\{v_{1}, v_{4}\right\}$ is a TDS for $G$ of cardinality less than $\gamma_{t}(G)$, a contradiction. So $\gamma_{t}(H)>\gamma_{t}(G)$ and thus $b_{t}(G) \leq$ $\sum_{i=1}^{4} \operatorname{deg}\left(v_{i}\right)-\left|E\left(G_{1}\right)\right|-2$. The sharpness follows by Proposition 2 .

Since the graph $G_{1}$ constructed in previous theorem has at least 3 edges, $b_{t}(G) \leq$ $\sum_{i=1}^{4} \operatorname{deg}\left(v_{i}\right)-5$. Also if $\operatorname{deg}\left(v_{i}\right)=\delta(G)$ for some $1 \leq i \leq 4$, then $b_{t}(G) \leq$ $3 \Delta(G)+\delta(G)-5$.

The following upper bound is also useful.

Lemma 7 Let $(x, y, z, w)$ be a path in a graph $G$ and let $\operatorname{deg}(w) \leq \operatorname{deg}(x)=$ $\operatorname{deg}(y)=\operatorname{deg}(z)=2$. Then

$$
b_{t}(G) \leq \operatorname{deg}(w)+1 \text {. }
$$

Proof By Propositions 1 and 2, we assume that $G \notin\left\{P_{4}, P_{5}, P_{6}, C_{4}, C_{5}\right\}$. Let $a \neq y$ be a vertex adjacent to $x$. 
Assume first that $\operatorname{deg}(w)=1$. Let $S_{1}$ be a $\gamma_{t}(G-a x-y z)$-set. If $\left|S_{1}\right|=\gamma_{t}(G)$, then $S_{1}-\{w, x\}$ is a TDS for $G$ of cardinality smaller than $\gamma_{t}(G)$, a contradiction. This implies that $\left|S_{1}\right|>\gamma_{t}(G)$ and hence $b_{t}(G) \leq 2=\operatorname{deg}(w)+1$.

Assume next that $\operatorname{deg}(w)=2$. Let $b \neq z$ be a vertex adjacent to $w$. If $\operatorname{deg}(a)=1$ or $\operatorname{deg}(b)=1$, then the situation is similar to the case when $\operatorname{deg}(w)=1$. Thus we may assume that $\operatorname{deg}(a) \geq 2$ and $\operatorname{deg}(b) \geq 2$. Let $S_{2}$ be a $\gamma_{t}(G-a x-y z-w b)$-set. If $\left|S_{2}\right|=\gamma_{t}(G)$, then $S_{2}-\{x, w\}$ is a TDS for $G$ of cardinality smaller than $\gamma_{t}(G)$. This implies that $\left|S_{2}\right|>\gamma_{t}(G)$ and so $b_{t}(G) \leq 3=\operatorname{deg}(w)+1$.

Open Access This article is distributed under the terms of the Creative Commons Attribution License which permits any use, distribution, and reproduction in any medium, provided the original author(s) and the source are credited.

\section{References}

1. Bauer, D., Harary, F., Nieminen, J., Suffel, C.L.: Domination alteration sets in graphs. Discrete Math. 47, 153-161 (1983)

2. Domke, G.S., Laskar, R.C.: The bondage and reinforcement numbers of $\gamma_{f}$ for some graphs. Discrete Math. 167(168), 249-259 (1997)

3. Dunbar, J.E., Haynes, T.W., Teschner, U., Volkmann, L.: Bondage, insensitivity and reinforcement. In: T. W. Haynes, S. T. Hedetniemi, P. J. Slater (eds.), Domination in Graphs: Advanced Topics, Marcel Dekker, New York, 471-489 (1998)

4. Fink, J.F., Jacobson, M.S., Kinch, L.F., Roberts, J.: The bondage number of a graph. Discrete Math. 86, 47-57 (1990)

5. Hartnell, B.L., Rall, D.F.: Bounds on the bondage number of a graph. Discrete Math. 128, 173177 (1994)

6. Haynes, T.W., Hedetniemi, S.T., Slater, P.J.: Fundamentals of Domination in Graphs, Marcel Dekker, New York, (1998)

7. Henning, M.A.: A survey of selected recent results on total domination in graphs. Discrete Math. 309, 32-63 (2009)

8. Hu, T., Xu, J.-M.: On the Complexity of the Bondage and Reinforcement Problems, Journal of Complexity 28 192-201, (2012). doi:10.1016/j.jco.2011.11.001

9. Kulli, V.R., Patwari, D.K.: The total bondage number of a graph, Advances in Graph Theory, Vishwa International Publication, 227-235 (1991)

10. Lu, Y., Xu, J.-M.: The p-Bondage Number of Trees, Graphs and Combinatorics 27:129-141 (2011). doi:10.1007/s00373-010-0956-3

11. Raczek, J.: Paired bondage in trees. Discrete Math. 308, 5570-5575 (2008)

12. Sridharan, N., Elias, M. D., Subramanian, V. S. A.: Total bondage number of a graph. Akce J. Graphs Combinator. 4, 203-209 (2007)

13. Teschner, U.: New results about the bondage number of a graph. Discrete Math. 171, 249-259 (1997) 